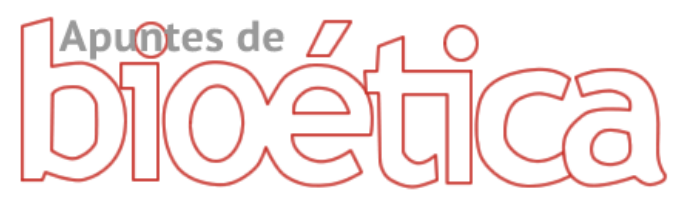

https://doi.org/10.35383/apuntes.v2i1.239

\title{
Prevención de la Medicalización de la Depresión: la Intervención del Psicólogo
}

\author{
Frauca Catalán Patricia ${ }^{1}$, López Guzmán José ${ }^{2}$
}

\section{INFORMACIÓN DEL ARTÍCULO RESUMEN}

Historia del artículo:

Recibido el 19 de marzo de 2019

Aceptado el 19 de julio de 2019

Palabras claves:

Medicalización

Depresión

Psicólogo

Antidepresivos

Equipo sanitario

Visión holística

En los últimos años se ha incrementado el consumo de fármacos antidepresivos. Entre las razones que llevan a ese aumento en el consumo de antidepresivos, están aquellas incluidas en la "medicalización", proceso por el que situaciones normales de la vida han pasado a ser tratadas desde el punto de vista médico como único remedio. Aunque los antidepresivos son de gran ayuda, no deben ser la única herramienta, ni la primera en todos los casos, a la que se recurra para el tratamiento de la depresión. En muchas situaciones, el ser humano necesita que le ayuden a racionalizar y canalizar los sentimientos que, en ciertas situaciones, pueden desbordarse. Es ahí donde el psicólogo tiene una labor fundamental, al evitar que una situación normal llegue al ámbito patológico. Por ello, una buena estrategia para paliar la medicalización de la depresión debe incluir una mayor presencia del psicólogo en el equipo sanitario.

\section{Prevention of medicalization of depression: The psychological intervention}

\section{ABSTRACT}

\section{Keywords:}

Medicalization

Depression

Psychologist

Antidepressant drugs

Healthcare team

Holistic vision
In recent years, the use of antidepressant drugs has increased. Amongst the reasons that have led to the increase in the use of antidepressants, are those included in the "medicalization" process, by which normal situations of life have been treated, as the only remedy, from a medical point of view. Although antidepressants are of great help, they should not be the only resource, nor the first resource in any situation, to be used for the treatment of depression. In many situations, human beings need assistance in order to rationalize and channel their emotions which, in certain situations, can

\footnotetext{
${ }^{1}$ Graduada en Farmacia. Estudiante de doctorado. Departamento de Farmacología y Toxicología. Facultad de Farmacia y Nutrición. Universidad de Navarra. Navarra España. E-mail: pfrauca@alumni.unav.es, pfrauca93@gmail.com ORCID: https://orcid.org/0000-0002-9822-2095

${ }^{2}$ Doctor en Farmacia. Profesor Titular. Departamento de Farmacología y Toxicología. Facultad de Farmacia y Nutrición. Universidad de Navarra. Navarra, España. Email: jlquzman@unav.es, ORCID: https://orcid.org/0000-0001-7308-3087
} 
easily get out of control. It is in these situations where a clinical psychologist has an important task of preventing a normal situation from turning into something considered to be pathological. Therefore, a good strategy to alleviate the "medicalization" of depression should include a greater presence of a psychologist in any healthcare team.

\section{Introducción}

De acuerdo con el Instituto Nacional de Estadística, en España 2,8 millones de personas sufren algún tipo de depresión (Instituto Nacional de Estadística, 2014), y la Organización Mundial de la Salud señala que esta es una de las primeras causas de discapacidad a nivel internacional (Organización Mundial de la Salud, 2017). Por su parte, la Agencia Española de Medicamentos y Productos Sanitarios indica que el consumo de fármacos antidepresivos se incrementó un 200\% entre los años 2000 y 2013 (Agencia Española de Medicamentos y Productos Sanitarios, 2015).

Este llamativo aumento de los casos de depresión y el uso de antidepresivos ha motivado a los investigadores a buscar la causa que explique dicho fenómeno. En principio, la prevalencia de la depresión podría deberse a una mayor incidencia de la patología, y eso justificaría el mayor consumo de antidepresivos. Sin embargo, hay datos que cuestionan la anterior afirmación y, por ello, se han buscado otros posibles factores que puedan influir como, por ejemplo, la incorrecta utilización de los fármacos antidepresivos. En este sentido, existen evidencias de que hay un problema de mal uso de estos medicamentos (McLennan \& Ulijaszek, 2018) (Perez Álvarez, 2007)

Entre las razones que llevan a un aumento en el consumo de antidepresivos por su incorrecta utilización, cabe destacar aquellas incluidas en el fenómeno de la "medicalización".
La medicalización ha sido definida como el proceso por el que situaciones normales de la vida han pasado a ser tratadas desde el punto de vista médico como único remedio, por ejemplo, la tendencia del hombre contemporáneo a convertir cualquier problema o deficiencia -incluso de tipo moral- en una patología.

Las siguientes páginas expondrán las características que definen la medicalización $y$, más concretamente, su relación con la depresión. A continuación, se ahondará en las consecuencias de la medicalización de esta enfermedad para, por último, proponer una serie de medidas que palien dicho problema público de salud. Entre ellas, cabe destacar el papel que el psicólogo clínico puede y debe desempeñar en el equipo sanitario a la hora de delimitar los casos de depresión de aquellos que no lo son (Cordero-Andrés et al., 2017).

\section{Metodología}

El presente trabajo se trata de una revisión bibliográfica descriptiva y evaluativa. Se ha realizado una búsqueda bibliográfica en la base de datos PubMed, con los términos en inglés "medicalization and depression" teniendo en cuenta aquellos artículos más relevantes sobre el tema y la bibliografía que se deriva de los mismos, también se han utilizado fuentes secundarias, libros divulgativos científicos y artículos de bioética no indexados. 


\section{Resultados y análisis}

\section{La medicalización}

El término "medicalización" comenzó a utilizarse mayoritariamente en la década de los 70 (Foucault, 1976) para designar aquellos procesos que buscaban transformar situaciones normales de la vida humana -por ejemplo, la menopausia, la calvicie, el duelo- en cuadros patológicos susceptibles de ser tratados desde un punto de vida médico (Chodoff, 2002). A pesar de que el concepto es relativamente reciente, se ha ido desarrollando y ha adoptado otras acepciones, como el recurso a la medicina para solucionar situaciones que no son realmente problemas de salud, sino que tienen origen antropológico (Conrad, Mackie, \& Mehrotra, 2010); el empleo de fármacos o procedimientos médicos para alcanzar ciertos estándares sociales -cirugía estética, dietas milagro- y mentales -estimulantes psíquicos, antidepresivos, tranquilizantes, etc.- (López Guzmán, 2013). A esto último se le ha llegado a denominar "cosmética psicofarmacológica" (Hernáez, 2007).

Uno de los tipos de medicalización se asocia a un problema antropológico y supone un riesgo moral para el ser humano ya que lo separa de su propio crecimiento personal, anestesiando con medicamentos las inquietudes vitales y dejando en manos de agentes externos la solución de problemas cruciales de la existencia. De esta manera, impide la maduración de la personalidad, por lo que tiene una especial incidencia en el desarrollo del sujeto y lo hace más vulnerable a la medicalización. Por otra parte, la medicalización tiene una repercusión en el ámbito sanitario al poder derivar en iatrogenia, que va a tener un impacto en la salud del sujeto afectado, de la salud pública y en la praxis del profesional sanitario. (Moré Herrero, 2013). Las anteriores apreciaciones tienen una gran relevancia en la valoración bioética de la actividad asistencial de la depresión.

\section{Incremento en el diagnóstico de la depresión y en el uso de psicofármacos}

Es un hecho constatable que, en los últimos años, se está produciendo un acrecentamiento en la cantidad de diagnósticos de depresión. Como ya se ha mencionado, cabría pensar que esto se debe a que hay más personas afectadas por la patología. Sin embargo, varios autores lo cuestionan (Ortiz Lobo \& Lozano Serrano, 2005); (Moloney, 2017), algunos sostienen que no se corresponde con la realidad (López Méndez \& Costa Cabanillas, 2015), y otras numerosas fuentes ponen en tela de juicio el enfoque dado al tratamiento de la depresión (Lawrence, Rasinski, Yoon, \& Curlin, 2015). Por otra parte, el constante aumento en el consumo de antidepresivos ha hecho pensar en ciertas causas no directamente relacionadas con la enfermedad. La más acuciante es la tendencia de la sociedad actual a interpretar las reacciones emocionales o anímicas ante una situación compleja -por ejemplo, la tristeza de un desamor, la pérdida de un ser querido, etc. como un problema médico. Se sabe que el estrés puede producir algunos tipos de somatización (taquicardias, dolores musculares, afecciones dermatológicas, digestivas, etc.). Esto no es más que la comprobación de que el ser humano es una unión entre lo psíquico y lo físico.

No obstante, el hecho de que exista una relación causa-efecto no significa que el tratamiento del síntoma, el efecto, solucione la causa. De esta manera, por ejemplo, en el caso de un duelo por la pérdida de un ser querido que no ha sido superada, habrá que tratar desde una perspectiva profesional la tristeza que provoca el duelo; en lugar de limitarse a 
recetar un antidepresivo como única solución al problema. Esto es algo que debe abordarse desde la perspectiva de la bioética, dado que proviene de una visión puramente materialista del hombre, con el fin de obtener un beneficio monetario o de poder, se ve afectada la dignidad del ser humano.

La industria farmacéutica se ha visto beneficiada en esta vulnerabilidad del ser humano contemporáneo ante la depresión. Durante muchos años, los grandes laboratorios han puesto en marcha diversas técnicas con el objetivo de incrementar sus ventas, sin tener en cuenta la repercusión de esto en la salud de la población. Entre ellas destaca la de ampliar el número de patologías a tratar por los medicamentos y la expansión de los límites de las enfermedades mediante una rebaja en los umbrales de diagnóstico, de forma que quedaría justificado indicar fármacos con mayor frecuencia. Esto último se trata de un hecho documentado, como se pone en evidencia en numerosos artículos, y la $\mathrm{V}$ edición del Manual Diagnóstico y Estadístico de los Trastornos Mentales (en adelante, DSM-V) que ha recibido numerosas críticas, por determinaciones como la de incluir la categoría de duelo como uno de los criterios de diagnóstico de la depresión (Sachdev, 2013); (Moynihan, Heath, \& Henry, 2002); (Tovar Bobo, Cerecedo Pérez, \& Rozadilla Arias, 2013); (Stolkiner, 2013). Un ejemplo de la considerada invención de enfermedades es el caso del Paxil ${ }^{\circledR}$ (paroxetina comercializada por la multinacional GlaxoSmithKline), presentado por el laboratorio para combatir la "ansiedad social", aprobado en origen para el tratamiento de la depresión en adultos(Le Noury et al., 2015).

Otro factor digno de considerar, en esta descripción de las causas que han incrementado el consumo de antidepresivos, consiste en que el sistema público de salud español ha delegado la responsabilidad del tratamiento a los especialistas en medicina de familia, recurriendo en muy pocas ocasiones a los profesionales de la salud mental por falta de recursos (López Méndez \& Costa Cabanillas, 2015). Los médicos de familia disponen de poco tiempo para atender a los pacientes, una media de siete minutos por consulta, que resultan insuficientes para un diagnóstico de depresión que requiere, cuanto menos, un estudio riguroso de las causas que han llevado al estado depresivo del paciente. Además, las herramientas diagnósticas que se pueden aplicar en siete minutos de consulta son aproximaciones poco precisas sobre la dolencia que podría sufrir el paciente. (Dowrick \& Frances, 2013).

La cuarta causa del empleo irracional de psicofármacos es una visión únicamente orgánica del ser humano, tanto por parte de los facultativos como de la sociedad en general y de los propios pacientes. Tampoco ayuda la cultura de la inmediatez, que busca soluciones rápidas a todos los problemas: "Todo esto se ve favorecido por la actitud de la sociedad, que, en gran proporción, está exenta de horizontes personales y se deja deslumbrar por la materialidad e inmediatez de los pretendidos efectos [de los fármacos]" (López Guzmán, 2013)

Otra dificultad inherente al tema de este artículo es que el diagnóstico y tratamiento de la depresión, sin el conveniente seguimiento, hace al paciente percibir la enfermedad como si fuera algo extrínseco, al modo de una infección, y en consecuencia no puede hacer nada por sí mismo para mejorar (Heyno, 2017) . Esto constituye un arma de doble filo: por un lado, significa una liberación para las personas que sienten el estigma propio de la enfermedad mental; pero, por otro, el sujeto puede llegar a pensar que solo los fármacos van a poner fin a su enfermedad cuando, aunque haya condicionantes biológicos bien referenciados, siempre se puede hacer algo por mejorar el 
estado de salud y cambiar los hábitos o el estilo de vida que ha conducido a ese estado depresivo.

Por último, la ambigüedad en la definición de la enfermedad depresiva resulta clave para encontrar un remedio a la medicalización, puesto que, según las actuales definiciones, es bastante fácil confundir una tristeza diaria con esta enfermedad; $y$, como se ha expuesto anteriormente, tanto la acepción como los criterios que aporta el DSM-V no colaboran en gran medida a solucionar el citado problema.

\section{La participación de otros profesionales en el tratamiento de la depresión: la intervención del psicólogo.}

El apartado anterior señalaba que la falta de profesionales de la salud mental en el sistema público puede entrañar una causa del aumento de diagnósticos de depresión, pues gran parte de ellos los realiza directamente el médico de familia en lugar de un especialista en salud mental. En consecuencia, la mayoría de los tratamientos comienzan con la prescripción de psicofármacos, sin que antes el paciente haya sido derivado a un psicólogo que determine la verdadera causa de esa depresión, además, el psicólogo podría realizar un conveniente cribado en los pacientes que acuden a consulta con motivo de algún problema de salud mental. (Sanz \& Paz GarcíaVera, 2017).

El psicólogo clínico es aquel que "aplica el conocimiento y las habilidades, las técnicas y los instrumentos proporcionados por la Psicología y ciencias afines a las anomalías, los trastornos y cualquier otro comportamiento humano relevante para la salud y la enfermedad, con el fin de evaluar, diagnosticar, explicar, tratar, modificar o prevenir estos en los diferentes contextos en que los mismos puedan
manifestarse"(Colegio Oficial de Psicólogos, n.d.). De acuerdo con esta definición, el psicólogo tiene la capacidad de que el paciente modifique comportamientos humanos relevantes para la salud o para la enfermedad.

Pese a que la repetición de comportamientos convierta al individuo en una persona depresiva, la evidencia dice que las terapias psicológicas son igual de efectivas que los fármacos antidepresivos tanto en la modalidad leve como en la moderada de esta enfermedad, (González-Blanch et al., 2018); (Jakobsen, Lindschou Hansen, Storebø, Simonsen, \& Gluud, 2011); (Hegerl et al., 2010) lo cual posee vital relevancia porque con ellas podrían evitarse una gran cantidad de daños iatrogénicos $y$, además, se proporcionaría al paciente una disposición activa frente a la cura de su dolencia. Asimismo, a la sociedad en general le resulta útil tener un concepto de la persona como un ser dual -de mente y cuerpo- y evitar así el reduccionismo biologicista (Stolkiner, 2013). Como es sabido, cualquier concepción antropológica del hombre que no tenga en cuenta la unión entre el alma y el cuerpo que se da en el mismo, es una visión que lleva a errores éticos y que atenta contra la dignidad de la persona.

Sin duda, no todas las causas de la depresión son médicas. Muchas de ellas tienen su origen en la forma en que el paciente se relaciona con las demás personas y afronta las dificultades vitales. La depresión es una enfermedad grave, pero parece que el uso del término se ha banalizado (Turkheimer, 2015). Ante esta situación, nos encontramos con un profesional que puede ser de gran ayuda: el psicólogo. Éste encarna la faceta más humanística de todo este entramado, y es necesario que, basándose en la ciencia y una visión trascendente del ser humano, ayude a las personas a superar diferentes situaciones existenciales. Ya que este profesional aporta un 
valor añadido, al no centrarse solo en la depresión y sus efectos, sino que también contempla muchas de las adversidades que comúnmente llevan a la depresión como, por ejemplo, problemas sociales, un núcleo familiar inestable, un trabajo estresante, una política injusta, carencias y heridas afectivas, etc. Es decir, las causas.

\section{Discusión}

La psiquiatría y la psicología se mueven en un campo de difícil comprensión. La ausencia de marcadores biológicos en ambas disciplinas complica en muchos casos el correcto diagnóstico del paciente $y$, por eso, a veces la patología depresiva se confunde con otros aspectos. Por ejemplo, que el sujeto se plantee preguntas existenciales ante la muerte de un ser querido forma parte de la experiencia de vivir. El psicólogo puede acompañar en ese momento con el fin de que la respuesta a esas cuestiones desemboque en una maduración de la personalidad. De esta manera, se procura no convertir una vivencia compleja como es el duelo en una situación traumática y que, por otra parte, no reciba más solución que un medicamento, algo que sería reprobable desde el punto de vista de la bioética.

Se han señalado distintas causas para justificar este aumento de personas diagnosticadas con depresión. Entre ellas se encuentran una mayor agresividad del entorno social, una menor capacidad de afrontar la adversidad por parte de las personas, la propensión a resolver cualquier problema -sea de carácter médico o no- con medicamentos, una manera de actuar propia de la ideología transhumanista (Postigo, s.f) o la falta de criterios de diagnóstico eficientes.

Llegados a este punto hay que partir de la realidad de que los antidepresivos ayudan en el tratamiento de la enfermedad, ya que estos mejoran la plasticidad neuronal y el estado de ánimo del paciente. De esta manera, después resultará más fácil aplicar terapias conductuales, así como modificar los hábitos o el estilo de vida que han llevado al sujeto al estado depresivo (Assareh, Sharpley, McFarlane, \& Sachdev, 2015). No obstante, se insiste en que los psicofármacos no deben ser la única herramienta, ni la primera en todos los casos, a la que se recurra para el tratamiento de la depresión ya que los fármacos son capaces de anestesiar a corto plazo algunas inquietudes vitales $y$, con ello, se promueve una generación de personas afectivamente inmaduras, ya que cada experiencia de sufrimiento supone una oportunidad para aprender una lección de valor incalculable, por lo que no sería éticamente correcto evitar a las personas cualquier sufrimiento sin tener en consideración una apreciación holística de la situación.

Bien es cierto que, a veces, ante una tragedia el ser humano tiende a dramatizar. Por eso en la mayoría de los casos necesita de alguien que le enseñe a racionalizar y canalizar los sentimientos que en ciertas situaciones pueden desbordarse. Es ahí donde el profesional de la psicología tiene una labor fundamental: evitar que una situación normal llegue al ámbito patológico. De ahí que para una aplicación responsable de los medicamentos antidepresivos sea de vital importancia la integración del psicólogo clínico en el equipo asistencial de atención primaria. El buen profesional de la psicología que demanda la sociedad debe tener una base de formación científica, una ética sobresaliente y una visión holística del ser humano -en el plano físico y trascendente-. En conclusión, una de las medidas para combatir la medicalización de la depresión consiste en fortalecer el equipo multidisciplinar - médicos, farmacéuticos, enfermeros,...- con la figura del psicólogo clínico que puede ayudar a la temprana 
detección de conductas que no son patológicas y aquellas que sí puedan llegar a serlo $y$, en ese caso, sean derivadas a otro especialista.

En definitiva, hay una gran responsabilidad en la detección de comportamientos y conductas depresivas que sobre todo en una etapa inicial- no se corresponden a la enfermedad mental y que, por ello, no deben ser medicalizadas. La labor de cribado del psicólogo clínico es necesaria por ser fuente de mejora para cualquier paciente afectado tanto por estados de ánimo alterados, como por depresión. En el primer supuesto [estado del ánimo alterado], la participación temprana del psicólogo tendrá la consecuencia positiva de ayudar a prevenir de forma temprana el uso irresponsable de medicamentos que conduzcan a la medicalización.

\section{Conclusiones}

El consumo de medicamentos antidepresivos ha aumentado considerablemente debido a varias causas, una de ellas es la denominada medicalización de la vida.

Como consecuencia de la subjetividad del sufrimiento mental y la ausencia de marcadores biológicos, el diagnóstico de la patología depresiva puede confundirse con otros aspectos de la vida, provocando la medicalización de procesos no patológicos.

Es importante el proceso de acompañamiento ante un evento estresante, para que ese sufrimiento desemboque en la maduración de la personalidad. Es perjudicial y un acto de mala praxis proporcionar tratamiento farmacológico si no es realmente necesario. Sin embargo, se podrá prestar el tratamiento, en ciertos casos y por breve espacio de tiempo, como coadyuvante del acompañamiento.
Integrar al psicólogo clínico en el equipo de atención primaria, puede ser una de las medidas más importantes para paliar el proceso de la medicalización de la depresión, como parte de una apreciación más holística de la salud del ser humano.

\section{Bibliografía}

Agencia Española de Medicamentos y Productos Sanitarios. (2015). Informe de utilización de medicamentos antidepresivos en España durante el periodo 2000-2013. Madrid. Recuperado de http://www.aemps.gob.es/informa/boletin Mensual/2012/noviembre

Assareh, A. A., Sharpley, C. F., McFarlane, J. R., \& Sachdev, P. S. (2015). Biological determinants of depression following bereavement. Neuroscience \& Biobehavioral Reviews, 49, 171-181.

Chodoff, P. (2002). The medicalization of the human condition. Psychiatric Services (Washington, D.C.), 53(5), 627-8.

Colegio Oficial de Psicólogos. (s.f.). Psicologia Clinica y de la Salud. Recuperado el 19 de Septiembre de 2018, de https://www.cop.es/perfiles/contenido/clinic a.htm

Conrad, P., Mackie, T., \& Mehrotra, A. (2010). Estimating the costs of medicalization. Social Science \& Medicine, 70(12), 19431947.

Cordero-Andrés, P., González-Blanch, C., Umaran-Alfageme, O., Muñoz-Navarro, R., Ruíz-Rodríguez, P., Medrano, L. A., CanoVindel, A. (2017). Tratamiento psicológico de los trastornos emocionales en atención primaria: fundamentos teóricos y empíricos 
del estudio PsicAP. Ansiedad y Estrés, 23(23), 91-98.

Dowrick, C., \& Frances, A. (2013). Medicalising unhappiness: new classification of depression risks more patients being put on drug treatment from which they will not benefit. $B M J, 347(f 7140), 1-9$.

Foucault, D. M. (1976). Historia de la medicalización. Educación Médica y Salud, 10(1), 152-169. f

González-Blanch, C., Ruiz-Torres, M., CorderoAndrés, P., Umaran-Alfageme, O., Hernández-Abellán, A., Muñoz-Navarro, R., \& Cano-Vindel, A. (2018). Transdiagnostic Cognitive-Behavioural Therapy In Primary Care: An Ideal Setting. Revista de Psicoterapia, 29(110), 37-52.

Hegerl, U., Hautzinger, M., Mergl, R., Kohnen, R., Schütze, M., Scheunemann, W., ... Henkel, V. (2010). Effects of pharmacotherapy and psychotherapy in depressed primary-care patients: a randomized, controlled trial including a patients' choice arm. The International Journal of Neuropsychopharmacology, 13, 31-44.

Hernáez, Á. M. (2007). La mercantilización de los estados de ánimo. El consumo de antidepresivos y las nuevas biopolíticas de las aflicciones. Política y Sociedad, 43(3), 43-56.

Heyno, A. (2017). Editorial 234. Psychodynamic Practice: Individuals, Groups and Organisations, 23(4), 331-335. https://doi.org/10.1080/14753634.2017.13 88606

Instituto Nacional de Estadística. (2014). Encuesta Europea de Salud 2014. Estado de Salud: Cifras absolutas. Salud Mental. Prevalencia de cuadros depresivos activos según sexo y relación con la actividad económica actual. Recuperado 19 de septiembtre 2018, de http://www.ine.es/jaxi/Datos.htm?path =/t1 5/p420/a2014/p01/l0/\&file=12012.px

Jakobsen, J. C., Lindschou Hansen, J., Storebø, O. J., Simonsen, E., \& Gluud, C. (2011). The effects of cognitive therapy versus "treatment as usual" in patients with major depressive disorder. PloS One, 6(8).

Lawrence, R. E. ., Rasinski, K. A. ., Yoon, J. D. ., \& Curlin, F. A. . (2015). Psychiatrists' and primary care physicians' beliefs about overtreatment of depression and anxiety. Journal of Nervous and Mental Disease, 203(2), 120-125.

Le Noury, J., Nardo, J. M., Healy, D., Jureidini, J., Raven, M., Tufanaru, C., \& Abi-Jaoude, E. (2015). Restoring Study 329: efficacy and harms of paroxetine and imipramine in treatment of major depression in adolescence. BMJ, 351(h4320), 1-16.

López Guzmán, J. (2013). Medicalización y farmacia comunitaria. Aula de La Farmacia: Revista Profesional de Formación Continuada, 9(95), 47-55.

López Méndez, E., \& Costa Cabanillas, M. (2015). ¿Somos todos enfermos mentales? Manifiesto contra los abusos de la psiquiatría. Papeles Del Psicólogo, 36(1), 74-76.

McLennan, A. K., \& Ulijaszek, S. J. (2018). Beware the medicalisation of loneliness. The Lancet, 391(10129), 1480.

Moloney, M. E. (2017). Sometimes, it's easier to write the prescription': physician and 
patient accounts of the reluctant medicalisation of sleeplessness. Sociology of Health \& IIIness, 39(3), 333-348.

Moré Herrero, M. A. (2013). Medicalización de la Vida. Abordaje de su Demanda en Salud Mental Medicalization of Life. Boarding its Demand in Mental Health. Clínica Contemporánea, 4(1), 45-54.

Moynihan, R., Heath, I., \& Henry, D. (2002). Selling sickness: the pharmaceutical industry and disease mongering. $B M J$ (Clinical Research Ed.), 324(7342), 886-91. Recuperado de http://www.ncbi.nlm.nih.gov/pubmed/1195 0740

Organización Mundial de la Salud. (2017). Depresión. Retrieved April 24, 2017, from http://www.who.int/mediacentre/factsheets /fs369/es/

Ortiz Lobo, A., \& Lozano Serrano, C. (2005). El incremento en la prescripción de antidepresivos. Atención Primaria, 35(3), 152-155.

Perez Álvarez, M. (2007). La Activación Conductual y la Desmedicalización de la Depresión. Papeles Del Psicólogo, 28(2), 99-110.

Sachdev, P. S. (2013). Is DSM-5 defensible?
Australian and New Zealand Journal of Psychiatry, 47(1), 10-11.

Sanz, J., \& Paz García-Vera, M. (2017). Ideas equivocadas sobre la depresión y su tratamiento, 38(3), 177-184.

Stolkiner, A. I. (2013). Medicalización de la vida, sufrimiento subjetivo y prácticas en salud mental. In Capítulo de libro. Compilador: Hugo Lerner Colección FUNDEP. Buenos Aires: Psicolibro. (En prensa). Buenos Aires. Recuperado de: http://www.trabajosocial.unlp.edu.ar/uploa ds/docs/stolkiner_2013_medicalizacin_de_la _vida__sufrimiento_subjetiv_2014.pdf

Tovar Bobo, M., Cerecedo Pérez, M. J., \& Rozadilla Arias, A. (2013). Ética y prevención de la medicalización. Semergen: Revista Española de Medicina de Familia., (7), 376-381.

Turkheimer, E. (2015). What do we want from a depression diagnosis? In K. S. Kendler \& J. Parnas (Eds.), Philosophical issues in psychiatry III: The nature and sources of historical change. (pp. 319-322). New York, NY: Oxford University Press. 